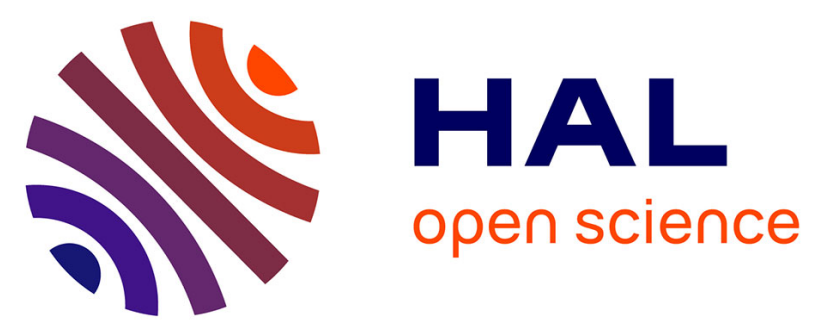

\title{
A local energy management system for solar integration and improved security of supply: The Nice Grid project
}

Andrea Michiorri, Robin Girard, Georges Kariniotakis, Christophe Lebossé, Sandrine Albou

\section{- To cite this version:}

Andrea Michiorri, Robin Girard, Georges Kariniotakis, Christophe Lebossé, Sandrine Albou. A local energy management system for solar integration and improved security of supply: The Nice Grid project. 3rd IEEE PES International Conference and Exhibition on Innovative Smart Grid Technologies (ISGT Europe), Dec 2012, Berlin, Germany. 6 p. - ISBN 978146732597, 10.1109/ISGTEurope.2012.6465667 . hal-00804442

\section{HAL Id: hal-00804442}

https://hal-mines-paristech.archives-ouvertes.fr/hal-00804442

Submitted on 12 Apr 2013

HAL is a multi-disciplinary open access archive for the deposit and dissemination of scientific research documents, whether they are published or not. The documents may come from teaching and research institutions in France or abroad, or from public or private research centers.
L'archive ouverte pluridisciplinaire HAL, est destinée au dépôt et à la diffusion de documents scientifiques de niveau recherche, publiés ou non, émanant des établissements d'enseignement et de recherche français ou étrangers, des laboratoires publics ou privés. 


\title{
A Local Energy Management System for Solar Integration and Improved Security of Supply: the Nice Grid Project
}

\author{
A. Michiorri, R. Girard, G. Kariniotakis, Senior Member, IEEE, C. Lebossé and S. Albou
}

\begin{abstract}
This paper describes Nice Grid, a demonstration project part of the European initiative Grid4EU. The project aims at developing a smart solar neighbourhood in the urban area of the city of Nice, France. The four year project started in November 2011 and will test the suitability of recent developments in distribution networks management for facilitating the connection of distributed renewable generators, improving the security of supply and let customers and other actors to provide network services. The idea behind Nice Grid is to combine controllable distributed electricity and thermal storage devices with forecasts of solar power production and load in a local energy management system. The paper, which represents a useful reference for the project, presents also a detailed overview of relevant European demonstration projects on Smart Grid.
\end{abstract}

Index Terms - Energy storage, Forecasting, Photovoltaic systems, Smart grids

\section{SUMMARY}

$\mathrm{N}$ ice Grid is a demonstration project led by ERDF, the main French distribution network operator, aiming at the development of a smart solar neighbourhood in Carros, in the urban area of Nice, France. The project, which started in November 2011 with a cumulative budget of $€ 38 \mathrm{M}$, will continue for four years and will test the suitability of recent development in distribution networks management for facilitating the connection of distributed renewable generators and for improving the security of supply. Nice Grid is partly sponsored by the ADEME under the initiative "Investissements d'Avenir" and the European Commission, through the 7th framework program. It is also part of Grid4EU, a €54M European initiative where six large demonstration projects are used to test technologies to

The work described in this paper is funded in part by the European Commission under the " $7^{\text {th }}$ Framework Programme" and ADEME under the program "Investissement d'Avenir"

A. Michiorri, R. Girard and G. Kariniotakis are with MINES ParisTech, CEP - Centre énergétique et procédés, CEP/Sophia Antipolis, BP 2071 rue Claude Daunesse 06904 Sophia Antipolis CEDEX, France (email : andrea.michiorri@mines-paristech.fr, robin.girard@mines-paristech.fr and georges.kariniotakis@mines-paristech.fr)

C. Lebossé is with ERDF Méditerranée, 6, allée Turcat Méry, 13008, Marseille, France. (email : christophe.lebosse@erdf-grdf.fr)

S. Albou is with the RTE / DES - Groupe Equilibre Offre Demande Court Terme, 9 Rue de la Porte de Buc, BP 561, 78005 Versailles cedex (email: sandrine.albou@rte-france.com) facilitate the integration of intermittent renewable resources and electric vehicles, and to test solutions for energy storage and energy efficiency.

Nice Grid is carried on by a consortium of ten companies and research institutions. Among them it is possible to mention ERDF the project leader, ALSTOM GRID a power system solutions provider, SAFT a battery manufacturer, EDF SA an electricity supplier, ARMINES a research institution and RTE the French transmission system operator.

The project is centred around a network energy management (NEM) system that will be tested on a network identified considering its characteristics and its ability to test the key objectives of the project. The chosen network, hosting a population of about 1500 residential and commercial customers, will also benefit from the infrastructure developed for the test. Two problems in particular are faced by the electric network in the region of Nice: the expected growth of solar power production at the distribution level and the possibility of disconnection from the transmission grid. The region is in fact at the end of an electric peninsula, with a great part of the load supplied by a single HV circuit. These two problems make the chosen network an ideal test-bed for technologies able to integrate large amounts of non firm distributed generators and to provide demand side management capabilities.

In order to achieve this, Nice Grid will use a combination of distributed energy storage, solar power production forecast and load forecast with a local energy management system. By optimising the charge-discharge cycle of the distributed storage facilities, the number of distributed generators disconnections due to the infringements of voltage constraints will be reduced. Furthermore the area will be able to reduce its load in case of planned outage. This will be facilitated by the use of demand side management of local loads and by the use of purpose-built probabilistic solar power forecast and load power forecast tools. The new smart meter infrastructure LINKY will also be used to provide daily detailed measurements of loads and photovoltaic production and will be used to deliver set points to controllable loads. The project will also demonstrate how different actors of the power system can provide network services. This will help to propose 
motivated regulatory changes for the future commercial implementation of the solutions tested.

A schematic representation of the system being developed and deployed is presented in Figure 1. The NEM (c) will be able to answer to requests of localized load variation coming from the DSO (b) also thanks to the PV production forecasts (d). The NEM makes use of offers of customers flexibility provided by the energy supplier or offers of network batteries flexibility (e). Once the optimal schedule is identified the controllable devices are informed using the smart meter infrastructure LINKY and other channels. It will be also possible for the TSO (a) to request flexibilities directly to the supplier for maximum of about 5MW. The different tests will be carried out in three areas: the islanding will affect a particular branch of the network in exam, the integration of PV will affect a larger portion of the network including the area used for islanding and the control of the demand will be tested on a larger area including the previous two.

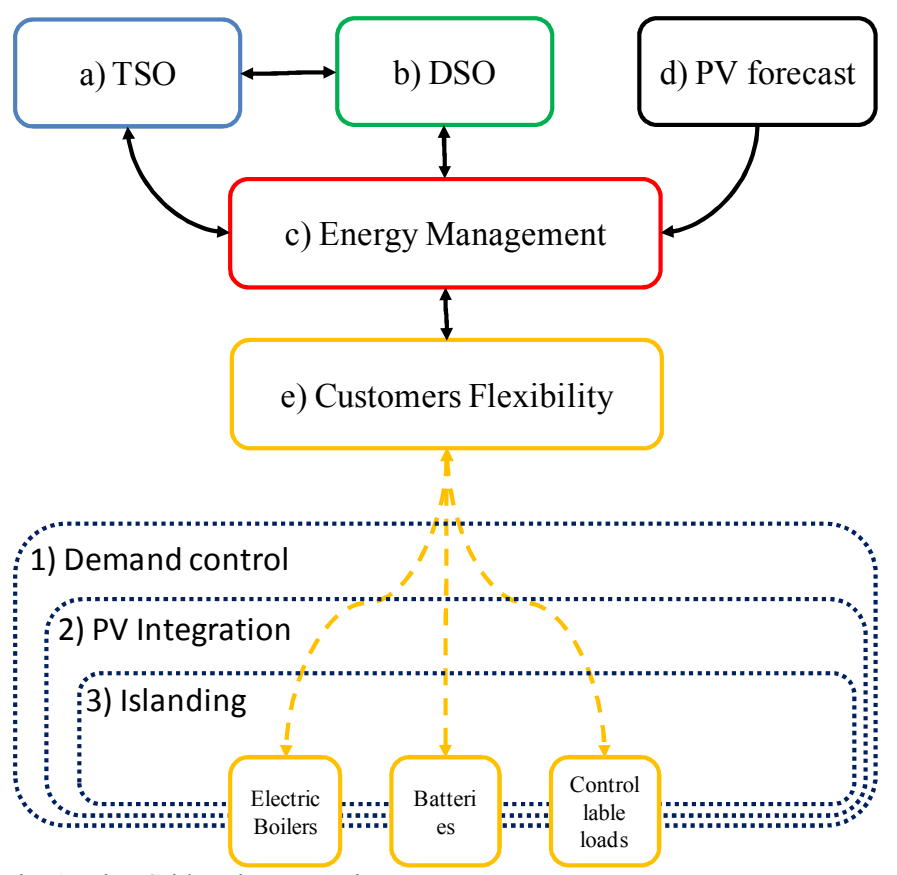

Fig. 1: Nice Grid project, overview

ERDF, the main French DSO is the leader of the project and will ensure the deployment and integration of the different components and technologies demonstrated in the project. It will also deploy its LINKY smart meter as the core communication vehicle between the local energy manager and the individual households. The intelligent control platform at the heart of the project is provided by ALSTOM, a manufacturer. The platform will manage and optimise in day ahead and in real-time all the eco-district's local energy resources within a low-voltage micro-grid. This will be done through a market based approach where aggregators can bid to offer flexibilities in each part of the network and in each time frame.

The contribution of EDF SA, the main electricity supplier in the French market, consists in implementing, with active participation from its clients, solutions to answer distributors' demands when facing network congestion issues. This can include encouraging clients to delay or suspend their power consumption when the balance between local offer and demand is non favourable. The company will also motivate its individual and professional customers to use thermal (hot water tanks) or electric (batteries) storage devices during photovoltaic high-production periods, to foster the integration of this production into the network. Effective visualization tools for photovoltaic consumption and production together with tailored economic incentives will allow EDF's clients involved in the Nice Grid demonstrator to contribute actively to optimise the local electric system. The relation with commercial customers is managed by NETSEENERGY, a subsidiary of EDF SA. In this project the company will develop modern communication protocols between utilities and business customers, a challenging task due to the diversity of local BMS systems and electricity uses.

The lithium-ion (Li-ion) batteries used in this project are provided by SAFT BATTERIES, a manufacturer. The batteries have an energy efficiency of around $95 \%$ and will be installed for a total capacity of about $2.7 \mathrm{MWh}$ throughout various voltage levels from the substation to the end user. At the substation level, a 1.1 MW / $512 \mathrm{kWh}$ unit will be installed in a standard 20-foot container. Batteries of the size of 100 $\mathrm{KW}$ will be installed in several locations of the distribution network to facilitate power flow management and to maintain voltages within limits. Finally, smaller batteries with a maximum size of $6 \mathrm{KW}$ will be installed in the premises of residential customers to enhance their demand response capabilities. Solar power production forecasts will be provided by ARMINES, an applied research institution. The forecasts will be based on commercial meteorological forecasts for the relevant parameters affecting solar power production and historical production datasets. The algorithm developed will ensure reliability, with the possibility of being deployed in other areas without difficult configuration. The main challenges of this task are represented by the stochastic behaviour of meteorological parameters and by the necessity to provide a forecast for the output of each single plant of the test network. ARMINES contributes also to the development of the control algorithm, facilitating the integration of stochastic forecasts into the system.

RTE, the French TSO, will use the project Nice Grid to test several scenarios of local demand curtailment, such as a dayahead reduction request in case of an expected ambient temperature reduction or a short term notice in case of outages. RTE will also participate in the specification of the interface between the transmission network and the distribution network in order to coordinate the decisions taken by the two operators to better integrate the local generation and the capacities of demand side management. Other partners include DAIKIN and WATTECO. DAIKIN, a multinational manufacturer, will provide demand response controls for air 
conditioning and heat pumps, whilst WATTECO, a French SME, will test the operational implementation of its communication technologies applied to demand response and the enPowerMe ${ }^{\mathrm{TM}}$ technology of disaggregation of consumption by use, in conjunction with demand response.

\section{BACKGROUND}

Innovations on smart grid related technologies developed during the last decades are now passing to a phase of technology demonstrations and deployments. This phase is witnessed by a series of demonstration projects in most developed countries. A comprehensive list of 219 demonstration projects in Europe is maintained by the European Commission Joint Research Centre [1] and further information on similar projects is found with national initiatives such as the French "Investissement d'Avenir" [2], the German "E-Energy" [3], the Italian "Active Networks and Smart Grids" [4], the British Low Carbon Network Fund [5] and the Spanish "FutuRed" [6]. From these sources, a total of 43 projects are considered relevant for the themes found in Nice Grid and are here briefly categorised according to the objectives of the demonstration of the project and the tools used.

Among pan-European collaborations, the projects Fenix [7], Microgrids [8], MoreMicrogrids [9], Open Meter [10], Address [11], Open Node [12] and Dispower [13] have developed or are developing ideas and technologies deployed in Nice Grid. In Belgium, the East Loop project [14] deals with the integration of renewable energy in an existing network with the use of active network management technologies. In France, Nice Grid follows three other pilot projects: MILLENER [15] on the use of batteries for the integration of renewable in islands, OPCO [16] on the use of demand response to improve the quality of supply in a weak network and PREMIO [17] to test demand response solutions for the integration of renewables.

In Germany, seven projects have been launched under the E-Energy initiative: eTelligence [18], e-Dema [19], MeRegio [20], Modellstadt Mannheim [21], Harz EE Mobility [22], Regenerative Modellregion Harz [23] and Smart Watt [24]. A common characteristic of these projects is the use of active participation of customers in the management of load flows and in markets, with the creation of local electricity markets such as in MeRegio and flexible tariffs in Modellstadt Mannheim. In Italy, eight different demonstrations have been started under the framework of the "Active Networks and Smart Grids" initiative [4]. A common characteristic of these demonstrations is the use of active network management for the integration of renewable energy at the MV network.

Eight demonstrations are being carried out in Spain under the framework of the FutuRed initiative or under the initiative of DSOs: in the project GAD [25] demand response solutions have been tested, in the projects DENISE [26] and ENERGOS [27] innovative control methods and the application of artificial intelligence have been tested, in VERDE [28] the integration of electric vehicles is tested and in REDES 2025 [29] solutions for network improvements on a medium long timeframe have been developed. Finally in the projects SCALA [30], STAR [31] and SMARTCITY [32] smart meters are being installed in a total of more than 190000 households and demand side management is used to facilitate the integration of renewables and electric vehicles on the distribution network. Finally in the United Kingdom two main deployment are carried out under the "Low Carbon Network Fund" initiative: in the LCL project [33] a combination of smart meters, electric vehicles and flexible tariffs is being tested, whilst in the CLNR project [34], flexible tariffs, interruptible contracts and smart meters are used to provide flexibility to the distribution network; finally, in the NINES [5] project demand side management is used for the integration of renewable in an islanded network.

These projects have been categorised in function of their objectives and the tools used to achieve them. The objectives considered are:

1. Integration of distributed generation

2. Peak reduction

3. Electric vehicles integration

4. Energy balance

5. Voltage or power flow constraints

6. Cyber security

Whilst the tools used are:

1. Demand side management

2. Generation output control

3. Smart meters

4. Flexible tariffs

5. Storage

6. Direct participation in electricity markets

7. Development of standards and guidelines

8. Use of forecasts.

The results of the classification are presented in TABLE I and TABLE II, where for each objective and each tool the number of occurrences in the projects analysed is reported. This shows that the most common objectives of the different projects are DER integration and peak load reduction, whilst the two most common tools used are demand side management and generation control. It is also important to consider that cyber security and forecasts are the least common objective and the least common tool used respectively.

TABLE I

Summary of the objectives of the projects considered

\begin{tabular}{|l|l|}
\hline \multicolumn{2}{|c|}{ Objectives } \\
\hline Distributed generation integration & 33 \\
\hline Peak reduction / Profile shaping & 18 \\
\hline Electric Vehicles Integration & 10 \\
\hline Energy balancing (Frequency) & 8 \\
\hline Voltage constraints & 5 \\
\hline Power flow constraints & 5 \\
\hline Cyber security & 2 \\
\hline
\end{tabular}


TABLE II

Summary of the tools used in the projects considered

\begin{tabular}{|l|l|}
\hline \multicolumn{2}{|c|}{ Tools } \\
\hline Demand side management & 24 \\
\hline Generation control & 23 \\
\hline Smart Meter & 16 \\
\hline Flexible tariff & 9 \\
\hline Energy storage & 7 \\
\hline Direct participation in energy market & 3 \\
\hline Standards and guidelines development & 3 \\
\hline Forecast & 1 \\
\hline
\end{tabular}

\section{ORIGINAL CONTRIBUTION}

The innovations of the project described in this work are also related to the scale of the demonstration and the number of customers involved. The objectives of the project, listed below and further expanded, are:

$A$. Provide services to the TSO and the DSO

$B$. Use of distributed Li-ion batteries

$C$. Forecasts of PV production and consumption

$D$. Optimise different sources of flexibility

$E$. Use of smart meters for monitoring and control

A. Enable individual customers to provide services to the TSO and the DSO, generating value for all the actors

The NEM system developed in Nice Grid will be able to transform the load flexibility of individual users into services for the TSO and DSO. The value generated can then be distributed among the different actors and, through the aggregators, to customers. The scale of the combined controlled loads will let the TSO to observe a variation in the demand of the substation with a reduction of at least $5 \mathrm{MW}$.

B. Use of distributed Li-ion energy storage to provide flexibility

Individual households' flexibility is obtained not only through demand side management, but also with the use of batteries. In this project, Lithium-ions batteries provided by SAFT are used. Li-ion batteries will also be installed on the network.

\section{Use of forecasts of $P V$ production and individual consumption}

A characteristic of Nice Grid is the use of predictions of solar power production for identifying potential LV grid voltage violations. This requires the forecast of the production at the level of individual producers on the low voltage network.

D. Use of an energy management system to optimize different sources of flexibility

The NEM will have to optimise the use of different form of flexibilities, balancing between the financial constraints and the comfort of the customers. The main sources of flexibility include: electric heating, electric boilers and Li-ion batteries. Commercial customers can provide flexibilities also in other ways depending on their process. These sources of flexibilities will be optimized in function of their availability, their cost and the maximization of customer's comfort.

\section{E. Use of smart meters for the monitoring and control of distributed devices}

Central to the Nice Grid project is the use of the smart metering infrastructure LINKY for managing the data transfer between the NEM and the individual household. The smart meter will be able to retrieve daily load curves from each household and production curves from each solar plant. It will be also used to dispatch orders to the individual controllable devices. The LINKY smart meter is being deployed in the French electric network and its integration in Nice Grid will make the solutions tested in this project easily deployed in other areas. Anyway batteries will be piloted by the aggregator with a dedicated infrastructure.

\section{PRELIMINARY RESULTS}

A preliminary study has been carried out to determine the precision of individual PV production forecasts with standard techniques. The objective is to identify optimal solution for the integration of forecasts into the NEM.

In this study, day-ahead forecasts of PV production have been calculated using a random forest regression. The forecasts have then been compared to the observations and the number of correct forecasts, missed forecast and false forecasts has been calculated. Two time series have been used in this work: the measured PV production of a PV plant connected to the network and the radiation forecasts that have been obtained by the European Centre for Medium-Range Weather Forecasts (ECWMF) [35]. Production measurements are recorded with a frequency of 10 minutes and the dataset used covers a period of nine months, between June 2011 and February 2012. The two time series are normalised and divided into two parts: the first part, composed by even days, is used to train the PV output forecast statistical model whilst the second, composed by odd days, is used to evaluate the model.

In this study the algorithm has been optimised to minimise the root square mean error (RSME) of the forecast at each time horizon. It is anticipated that in Nice Grid, one or more specific cost functions will be used for the training of the model. For the integration of PV production, the algorithm will aim at minimising the error in high production hours. It is also considered the possibility of assigning a higher weight to the underestimation PV production. PV Underestimation can in fact lead to the disconnection of the inverters, with a higher cost for the system respect to the simple de-optimisation of the batteries. On the other side, in the case of islanding, the goal of the model is to minimise the error in the estimation of the total energy produced by PVs in the day or in the specific timeframe of the outage.

The performance of the forecast is measured considering the number of high production events (called simply "events" below) correct forecasts, the number of missed events and the 
number of false forecasts. In this case an event is defined as an hour when the maximum measured or forecasted PV production is higher than $85 \%$ of the rated power. In Figure 2 the comparison between forecast and measured PV production for two days is shown. In case A, PV production follows a regular pattern that is well predicted by the forecast algorithm. In case $B$, probably because of the presence of clouds, the forecast algorithm predict a lower overall output with a more irregular pattern, but the observed PV output is considerably higher in the first part of the day and lower in the second part, whilst maintaining a correct estimation of the total daily production.
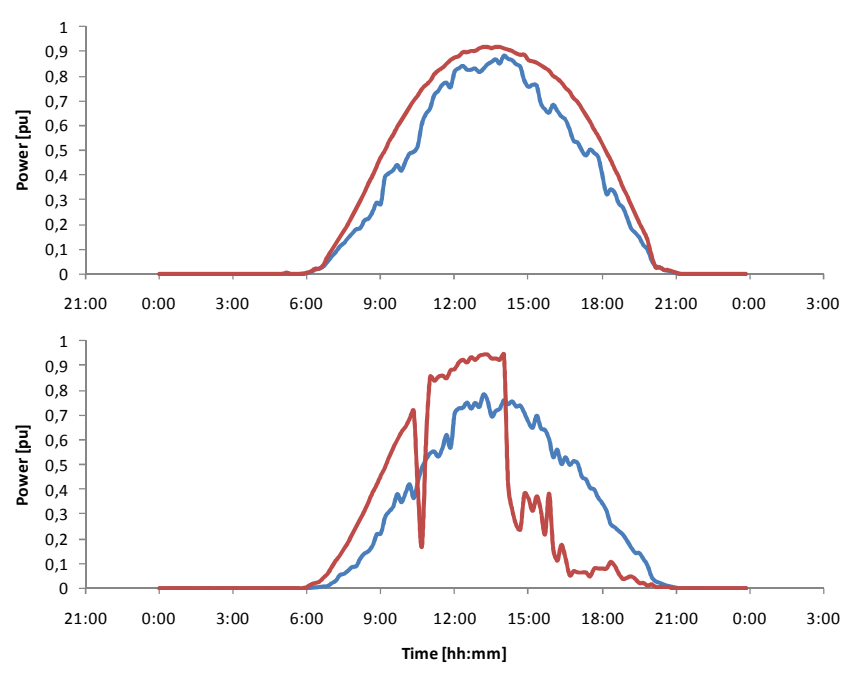

- Predictions —observations

Fig. 2. Dimensionless forecast and observed PV production for the day 27/06/2011 in case A and for the day 03/07/2011 in case B. Normalisation with the installed capacity of the PV plant

Occurrences where events are correctly forecasted have been recorded along with occurrences where false events where forecasted and where events were not forecasted. The results are summarised in TABLE III, where the percentage of occurrence respect to the effective observed events is reported. It can be seen that the algorithm is able to identify the $63 \%$ of the observed events, and that missed events are about the $42 \%$ more likely than false forecast events.

TABLE III

Precision of day-ahead PV production forecasts

\begin{tabular}{|l|c|}
\hline \multicolumn{1}{|c|}{ Parameter } & $\begin{array}{c}\text { Relative value on occurred } \\
\text { events [\%] }\end{array}$ \\
\hline Correct forecasts (hit ratio) & $63 \%$ \\
\hline Missed forecasts & $37 \%$ \\
\hline False forecasts & $26 \%$ \\
\hline
\end{tabular}

\section{CONCLUSIONS}

This paper presented the Nice Grid project, a demonstration of smart grid technologies in the urban area of Nice, France. The project, part of the European initiative Grid4EU, started in November 2011 will last four years. The objective of the project is to test for commercial use a series of concepts developed and tested in previous works. Nice Grid is centred on a local network energy management platform that will allow individual users to provide services to the TSO and the DSO through the intermediation of an aggregator. The platform will optimize the use of different sources of flexibility such as Li-ion batteries, electric heating and boilers, with a market-based approach. Central to the system will be the use of forecasts of PV production and loads on the low voltage network. The smart metering system LINKY will be used to monitor and control active loads in residential buildings.

The project is part of a series of development or demonstration projects are being carried out in the area of smart grids in Europe. A total of 43 projects have been considered relevant for Nice Grid and have been analysed and classified. The paper presents an overview of the results of the analysis, where it is shown that distributed generation integration with demand side management is the most common application deployed, whilst the testing of cyber security solutions and the use of forecasts are the less common. The paper presents also preliminary results on the works on the integration of PV production forecasts in the NEM system. The ability of standard PV production forecast statistical methods to identify hours of high production was verified. The algorithm tested presented a hit ration of $63 \%$, with missed forecasts the $43 \%$ more likely than false forecasts. Work is ongoing to improve the performance o the algorithm and to develop a strategy able to maximize the benefit of probabilistic forecasts in the NEM.

\section{ACKNOWLEDGEMENTS}

The authors wish to thanks the personnel of ERDF, ALSTOM, EDF SA, RTE, WATTECO, DAIKIN and SAFT BATTERIES for the support given.

\section{REFERENCES}

[1] V. Giordano, F. Gangale, G. Fulli, M. Sánchez Jiménez, "Smart Grid projects in Europe: lessons learned and current developments", JCR-IE, 2011, [Online]. Available: http://ses.jrc.ec.europa.eu/

[2] ADEME - Investissement d'Avenir, 2011, [Online]. Available: http://www2.ademe.fr/

[3] E-Energy, [Online]. Available: http://www.e-energy.de

[4] P. Pelacchi, F. Pilo, R. Turri, "Ammissibilità e graduatoria di merito delle istanze presentate ai sensi della deliberazione ARG/elt 39/10, relative a progetti pilota comprendenti sistemi di automazione, protezione e controllo di reti attive MT (smart grids)", 2011

[5] OFGEM - Low Carbon Network Fund, 2011, [Online]. Available: http://www.ofgem.gov.uk/

[6] FutuRed platform, 2011, [Online]. Available: http://www.futured.es/

[7] Project Fenix, 2011, [Online]. Available: www.fenix-project.org.

[8] Microgrids Project, 2011, [Online]. Available: http://www.microgrids.eu/micro2000/

[9] MoreMicrogrids Project, 2011, [Online]. Available: http://www.microgrids.eu/

[10] Open Meter Project, 2011, [Online]. Available: http://www.openmeter.com

[11] Address Project, 2011 [Online]. Available: http://www.addressfp7.org/

[12] Open Node Project, 2011, [Online]. Available: http://www.opennode.eu

[13] Dispower Project, 2011, [Online]. Available: http://www.iset.unikassel.de/dispower_static/ 
[14] O. Durieux, et al, "Smart grid technologies feasibility study: increasing decentralized generation power injection using global active network management", CIRED 2011, Frankfurt.

[15] Project MILLENER, 2011, [Online]. Available: http://www2.ademe.fr/

[16] EDF bleu ciel - Project OPCO, 2011, [Online]. Available: http://www.unebretagnedavance.fr

[17] Project Premio, 2011, [Online]. Available, http://www.projetpremio.fr/

[18] eTelligence, 2011, [Online]. Available: http://www.etelligence.de

[19] E-Dema, 2011, [Online]. Available: http://www.e-dema.de.

[20] Minimum Emission Region, 2011, [Online]. Available: http://www.meregio.de

[21] Modellstadt Mannheim, 2011, [Online]. Available: http://www.modellstadt-mannheim.de

[22] Harz EE Mobility, 2011, [Online]. Available: https://www.harzeemobility.de

[23] Regenerative Modellregion Harz, 2011, [Online]. Available: http://www.regmodharz.de

[24] Smart Watt, 2011, [Online]. Available: http://www.smartwatts.de

[25] Project GAD, 2012, [Online]. Available: http://gad.ite.es

[26] Project DENISE, 2012, [Online]. Available: http://www.cenitdenise.org/

[27] Project ENERGOS, 2012, [Online]. Available: $\mathrm{http}: / /$ innovationenergy.org/energos/

[28] Project Verde, 2012, [Online]. Available: http://www.cenitverde.es

[29] Project Redes 2025, 2012, [Online]. Available: http://www.redes2025.es/

[30] "Gas Natural Fenosa - una apuesta decidida por la eficiencia energetica", Ejecutivos - Istituciones y Empresa, 2011

[31] Project STAR, 2012, [Online]. Available: https://www.iberdrola.es/

[32] Project Smartcity, 2012, [Online]. Available: www.smartcitymalaga.es/

[33] Low Carbon London, 2011, [Online]. Available: http://lowcarbonlondon.ukpowernetworks.co.uk/

[34] Customer led network revolution, 2011, [Online]. Available: http://www.networkrevolution.co.uk/

[35] European Centre for Medium Range Weather Forecast, 2011, , [Online]. Available: www.ecmwf.int

\section{BIOGRAPHIES}

Andrea Michiorri recieved an MEng in Mechanical Engineering at the University of Rome "La Sapienza » and a PhD from the School of Engineering of Durham University (UK). After an experience in industry he is currently Research Engineer at the Centre of Energy and Processes of the Ecole des Mines de Paris. His research interests include the integration of distributed generation and the use of artificial intelligence in power systems.

Robin Girard received a Master's degree (2004) in Computer Science and Applied Mathematics from INPG in Grenoble, France and a PhD degree (2008) in applied Mathematics from University Joseph Fourier in Grenoble. $\mathrm{He}$ is currently a Research Engineer at the Centre of Energy and Processes of the Ecole des Mines de Paris. His research interests include wind and solar power forecast, optimisation in planning of energy production and spatiotemporal patterns of renewable power production in Europe.

George Kariniotakis (S'95 - M'02- SM'11) was born in Athens, Greece. He received his Production and Management engineering (1990) and M.Sc. (1992) degrees from the Technical University of Crete, Greece and his Ph.D. degree from Ecole des Mines de Paris in 1996. He is currently with the Centre of Energy and Processes of MINES ParisTech as a senior scientist and head of the Renewable Energies and SmartGrids Group. He is Senior Member of IEEE. His research interests include among others renewable energies, distributed generation and artificial intelligence.

Christophe Lebossé obtained a degree in Engineering at Ecole Centrale de Paris en 1986. He joined then EDF in 1989 and its subsidiary ERDF in 2007 where he worked on the design and management of the French distribution network. From 2011 he is project manager of Nice Grid for ERDF, where he contributes with his experience on the existing power system to the development of future electric networks.

Sandrine Albou received a MEng in Applied Mathematics from Ecole Supérieure d'Electricité (Supélec). In 2006, she joined the R\&D Department of RTE and worked on tools and methods of electricity consumption forecasts.
She is now Smart Grids Project Manager and she works on studies relative to the demand-supply balance. 